\title{
Abnormal Congenital Peritoneal Bands: A Retrospective Cadaveric Study
}

\section{ABSTRACT}

Introduction: Peritoneal folds are double layered peritoneal membranes produced during reflexion of peritoneum from viscera to the body wall or between adjacent viscera. They are named as ligaments, mesenteries, omentum etc. Sometimes during the course of development some abnormal peritoneal folds develop, which are of surgical importance because these folds or bands may constrict a part of intestine leading to obstruction and gangrene. These sometimes produce peritoneal recesses, which are the potential sites for internal herniation.

Aim: To study abnormal peritoneal folds in cadavers.

Materials and Methods: This was a descriptive study, conducted on 40 cadavers during August 2010-July 2020. Abdominal cavity was dissected and carefully observed for organisation of viscera and peritoneal reflexions in the abdominal cavity.
Results: Out of 40 cadavers, congenital abnormal peritoneal bands were found in four cases and two cases had multiple peritoneal bands. Three cases had associated congenital abnormalities of the gut. One case of nonrotation of gut was observed, where small intestine was situated on the right side and colon on the left side. In this case, the ascending colon was fixed by a peritoneal band to right upper abdominal wall. In the second case, there was incomplete fusion of descending mesocolon. The mobile segment of descending colon was fixed by an abnormal peritoneal band. In the third case, there was absorption of pelvic mesocolon.

Conclusion: Though the cause of development of abnormal peritoneal band is not very clear, this study shows that they may be present in cases of abnormal rotation or abnormal peritoneal fixation of gut.

Keywords: Intestinal obstruction, Internal herniation, Peritoneal recess, Rotation of gut

\section{INTRODUCTION}

Peritoneum is the most complexly arranged and largest serous membrane in the human body. During embryogenesis, all the abdominal viscera project into the cavity of peritoneum and take the layer of peritoneum along with them. The complex arrangement of visceral peritoneum results in formation of peritoneal folds which are named as ligaments, mesenteries, omenta etc. These folds are routes for blood vessels and lymphatics to reach the intraperitoneal organs from retroperitoneum. These peritoneal folds also suspend various viscera to support them [1]. Some avascular folds can be a connecting link between various viscera. Such folds create peritoneal recesses which are potential pockets for abscess formation [2]. It also provides a pathway for the spread of disease processes and sometimes limiting space for the spread of diseases [3,4].

Abnormal peritoneal bands may be formed due to abnormal embryological development or rotation of viscera or as a pathological process [5]. Such peritoneal bands may result in kinking of intestinal structures, leading to intestinal necrosis. They create fossae or recesses of the peritoneal cavity. These recesses thus produced are of clinical interest because a segment of intestine may enter one and may be a site of internal hernia [6]

Knowledge of these abnormal peritoneal folds is necessary for gastroenterologist and radiologist to avoid error or confusion in diagnosis and surgery. It also helps anatomists to understand the complex development and rotation of the viscera of the gastrointestinal system. Various abnormal folds have been reported in the previous studies. Ashaolu $\mathrm{JO}$ et al., in a series of study on 40 cadavers, observed 35\% (14 out of 40) occurrences of the cystoduodenal ligament [6]. Erginel B et al., in their study on 14 cases of intestinal obstruction due to congenital peritoneal bands have reported that they joined adjacent parts of intestine, most common being, terminal ilium to ascending colon (50\%) [7]. The present study was conducted to find the prevalence of abnormal peritoneal bands, common sites of occurrence, attachments, their association with other congenital abnormalities of gastrointestinal tract and to compare them with the findings of previous studies.

\section{MATERIALS AND METHODS}

This was a descriptive retrospective study from the documents of dissection conducted on 40 cadavers, allotted for first year MBBS study, in the Department of Anatomy during August 2010-July 2020. The precise age, medical history or the cause of death of the cadavers could not be ascertained. To exclude postoperative adhesion bands, cadavers with surgical scar on the abdominal wall were excluded from the study (20018/Dt-25-09-2020/IFO22/20). Abdominal cavity was dissected and carefully observed for various peritoneal folds along with position of abdominal viscera. A descriptive data analysis was done for each case where abnormal peritoneal folds were found.

\section{STATISTICAL ANALYSIS}

A descriptive data analysis was done for each case where abnormal peritoneal folds were found.

\section{RESULTS}

Abnormal congenital peritoneal folds were found in four cadavers out of 40 cadavers (10\%). Out of these four cases multiple peritoneal bands were observed in two cases. Three cases had associated abnormalities of gastrointestinal tract.

\section{Case 1}

It was a case of nonrotation of the gut in which an abnormal peritoneal band extended from the upper end of ascending colon to the inferior surface of the right lobe of liver and then to the peritoneum of the right upper lateral abdomen. Though normally in cases of nonrotation hepatic flexure and transverse colon are not formed, the angulation produced by the attachment of this peritoneal band in the present case of nonrotation, produced a so-called hepatic flexure and transverse colon. This band kept the colon adherent 
to the inferior surface of liver and had to be separated to make the colon free from the liver [Table/Fig-1,2].
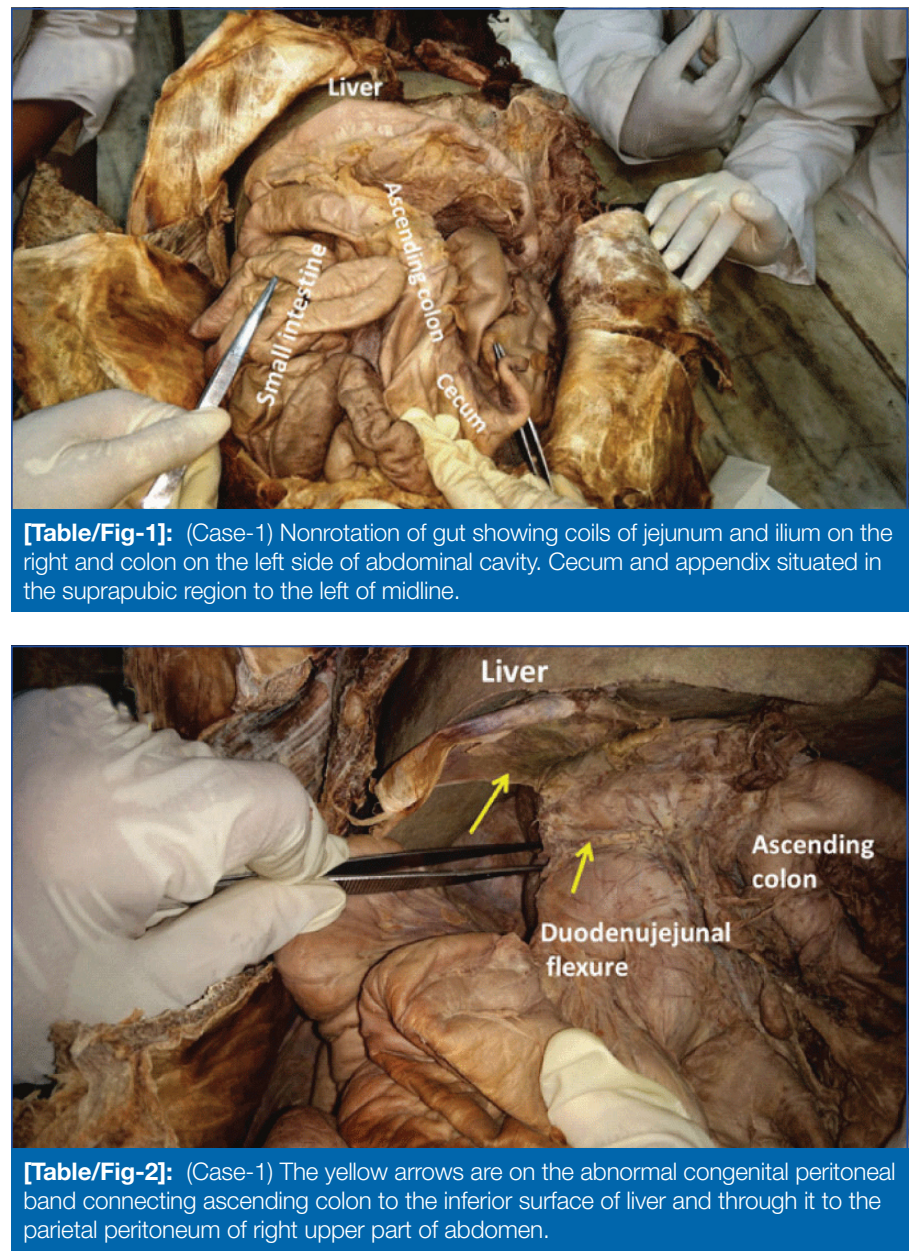

\section{Case 2}

In this case, two abnormal peritoneal bands were found in the supracolic compartment connecting duodenum to liver and gallibladder. One of the peritoneal bands was abnormally reflected from the inferior surface of right lobe of liver to the junction of second and third part of duodenum (hepatoduodenal ligament). The other band extended from gallbladder to the third part of duodenum (cystoduodenal ligament). Both the bands were double layered membranous structures covering gallbladder and duodenum. There were no neurovascular structures within it. These accessory peritoneal folds were differentiated from the peritoneal adhesions by tracing the two layers around the viscera connected by them [Table/Fig-3].

\section{Case 3}

This was a case of abnormal peritoneal fixation of colon where the sigmoid colon had become retroperitoneal by absorption of its mesentery. In this case an abnormal peritoneal band was found, connecting the inferior border of right lobe of liver to the right colic flexure (hepatocolic ligament). This was an accessory peritoneal band which did not have any neurovascular structures and was a double layered structure [Table/Fig-4].

\section{Case 4}

This was also a case of abnormal peritoneal fixation of colon, where a large segment of descending colon had become intraperitoneal by persistence of descending mesocolon and the pelvic colon became retroperitoneal by absorption of pelvic mesocolon [Table/Fig-5]. Proximal and distal ends of the descending colon were retroperitoneal and intermediate part was suspended by mesocolon attached to the left lateral margin of vertebral column. The intraperitoneal segment of descending colon occupied the left hypochondrium, epigastrium and umbilical region of abdomen and its original position, the left flank was empty. The mobile segment

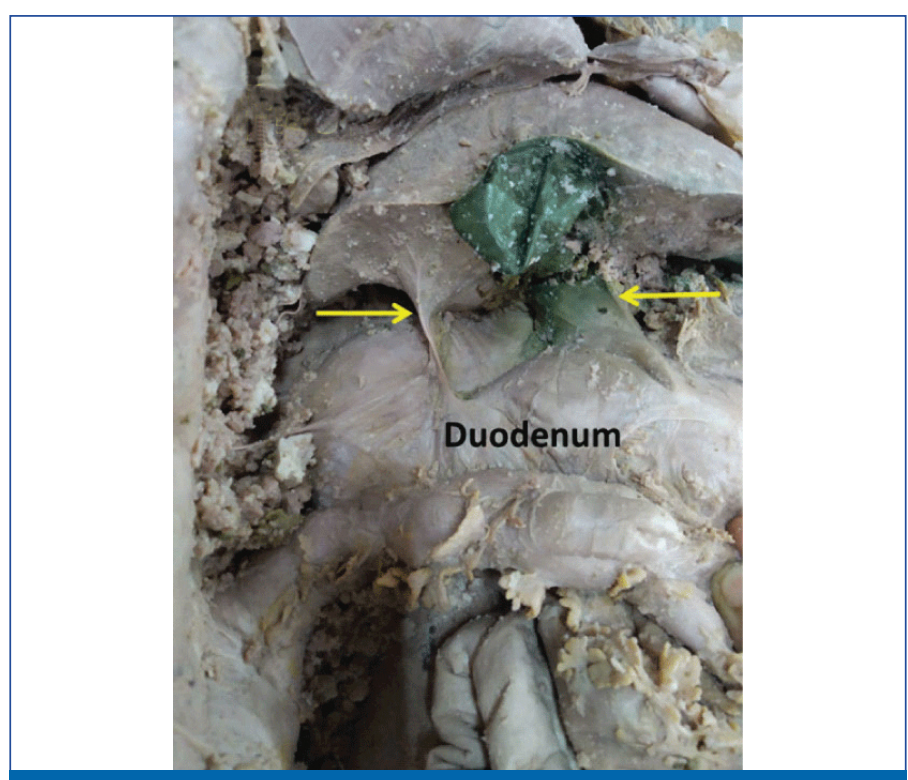

[Table/Fig-3]: (Case-2) Peritoneal band connecting right lobe of liver to junction of second and third part of duodenum (the yellow arrow on the left). The second band connecting gall bladder to third part of duodenum (the yellow arrow on the right).

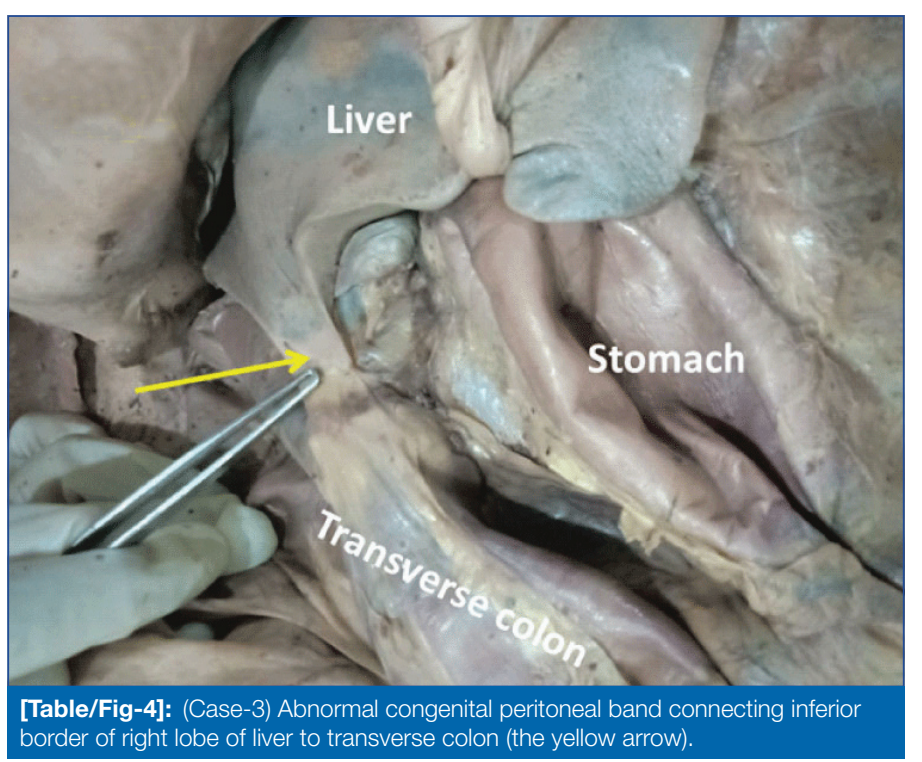

of descending colon was kept fixed in this particular position by an abnormal peritoneal band connecting it to the left colic flexure. This band was a vascular band carrying branches of the left colic artery through it and was responsible for pulling the descending colon out of its original position. Another peritoneal band was found in the site, where the peritoneal relation of descending colon abruptly changed from retroperitoneal to intraperitoneal. This band was a double

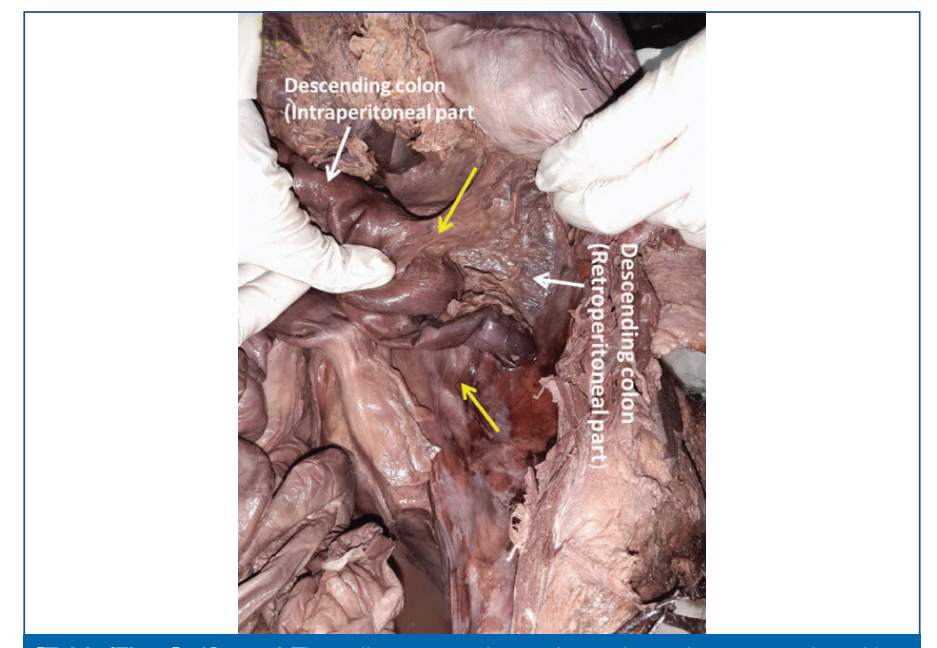

[Table/Fig-5]: (Case 4) The yellow arrow shows the peritoneal recess enclosed by the abnormal peritoneal band. 
layered reflexion of peritoneum with a deep peritoneal recess under it. In addition to these two bands, a loop of terminal ileum was also found to be connected by an abnormal peritoneal band.

\section{DISCUSSION}

The exact mode of origin of abnormal congenital peritoneal bands is doubtful. These have been attributed to errors in development, mechanical traction by the gut and even linked with evolution of upright posture. The most common type of anomalous folds is prolongation of lesser omentum to the right of usual site of epiploic foramen. These are peritoneal folds extending from gallbladder to the superior part of duodenum, called cystoduodenal ligament or in front of the superior part of duodenum to the greater omentum or right colic flexure or from inferior surface of right hepatic lobe to the right colic flexure, called hepatocolic ligament [8].

Number of case reports is available on cystoduodenal, cystoduodenocolic and hepatocolic ligaments. Sharma NA et al., reported two separate peritoneal bands in a cadaver, cystoduodenal and hepato-duodeno-colic ligaments [2]. Nayak SB et al., reported an omento-cystic peritoneal fold in an adult male cadaver which extended upwards from the right end of the greater omentum to the gallbladder [9].

Nayak SB et al., reported another case of cysto-duodenocolic ligament where an abnormal peritoneal band joined the second part of duodenum and transverse colon to the gall bladder causing compression to both duodenum and colon [5]. In the present study, in second and third case, similar type of peritoneal bands were found connecting different parts of duodenum and transverse colon to the liver and gallbladder [Table/Fig-3,4].

Peritoneal bands are also formed due to abnormal disposition of intestines and mesentery resulting from errors in rotation of gut. Improper fusion of mesenteries may constrict or angulate some part of the gut. Wakefield EG et al., in their study on intestinal obstruction due to mesenteric band, in association with failure of intestinal rotation, emphasised the role of such bands in producing obstruction. They regarded these congenital mesenteric bands, which are typically attached to the lower portion of duodenum, the upper portion of jejunum or the so-called transverse colon, as having a definite function during the period of development, and never resulted from adhesion. These bands have been described as thickened areas of mesentery which may produce angulation of a part and thus produce intermittent intestinal obstruction [10]. Appaji AC et al., reported a case of nonrotation where a large band extended from the ascending colon to the left colic flexure. Another band extended from the descending colon to the lateral abdominal wall on the left side [11]. In the present study, the first case was a case of nonrotation. The disposition of intestines in the present case of nonrotation was little different than the usual cases of nonrotation because of a peritoneal band connecting ascending colon to the liver and through the liver to the parietal peritoneum of right upper abdomen. In nonrotation, caecum and appendix lie just above the pubic symphysis and usually to the left of midline. From this position the colon loops upward towards its point of fixation at the splenic flexure, and without the interposition of a true transverse colon, turns downward to form the descending colon. Thus, ascending and descending colons form two limbs of the loop of large intestine running parallel to each other. But in the present case, the upper end of ascending colon was fixed to the inferior surface of right lobe of liver and adjacent part of parietal peritoneum by a peritoneal band producing an angulation which appeared like hepatic flexure. The segment of colon from this point up to splenic flexure looked like transverse colon. Normally, hepatic flexure develops only in third stage of rotation when the caecum descends from the subhepatic position by the growth of large intestine [12]. In the present case, the rotation gut was arrested in first stage [Table/Fig-1,2].
Abnormal peritoneal bands also occur due to anomalies in second and third stage of rotation. The main events of third stage of rotation are fixation of colon and formation of root of mesentery. There is lengthening of colon with the formation of hepatic flexure to differentiate the ascending from transverse colon [12]. The chief anomalies of third stage are subhepatic caecum or mobile caecum and improper fusion of mesentery [12]. Due to abnormal rotation of midgut, if the caecum has remained in the right upper quadrant, it may become fixed in that position by peritoneal attachments passing to the right, the so-called Ladd's bands. These may compress the underlying duodenum and give rise to duodenal stenosis [1]. Saxena $P$ et al., reported an abnormal peritoneal band fixing the caecum to the lateral abdominal wall crossing the duodenum, the Ladd's band [13]. Improper fusion of ascending mesocolon, especially the first part gives rise to mobile caecum. Incomplete fusion may involve variable length of either ascending or descending mesocolon or both. An incomplete fusion may raise a peritoneal fold and develop a peritoneal pocket behind it which is a potential site for retrocolic or paracolic hernia [12]. In the present study, the fourth case was a case of incomplete fusion of descending mesocolon raising a peritoneal fold at the site where the peritoneal relation changed from retroperitoneal to intra peritoneal. Behind this peritoneal fold was a deep peritoneal recess which could have been a site for internal herniation [Table/Fig-5,6].

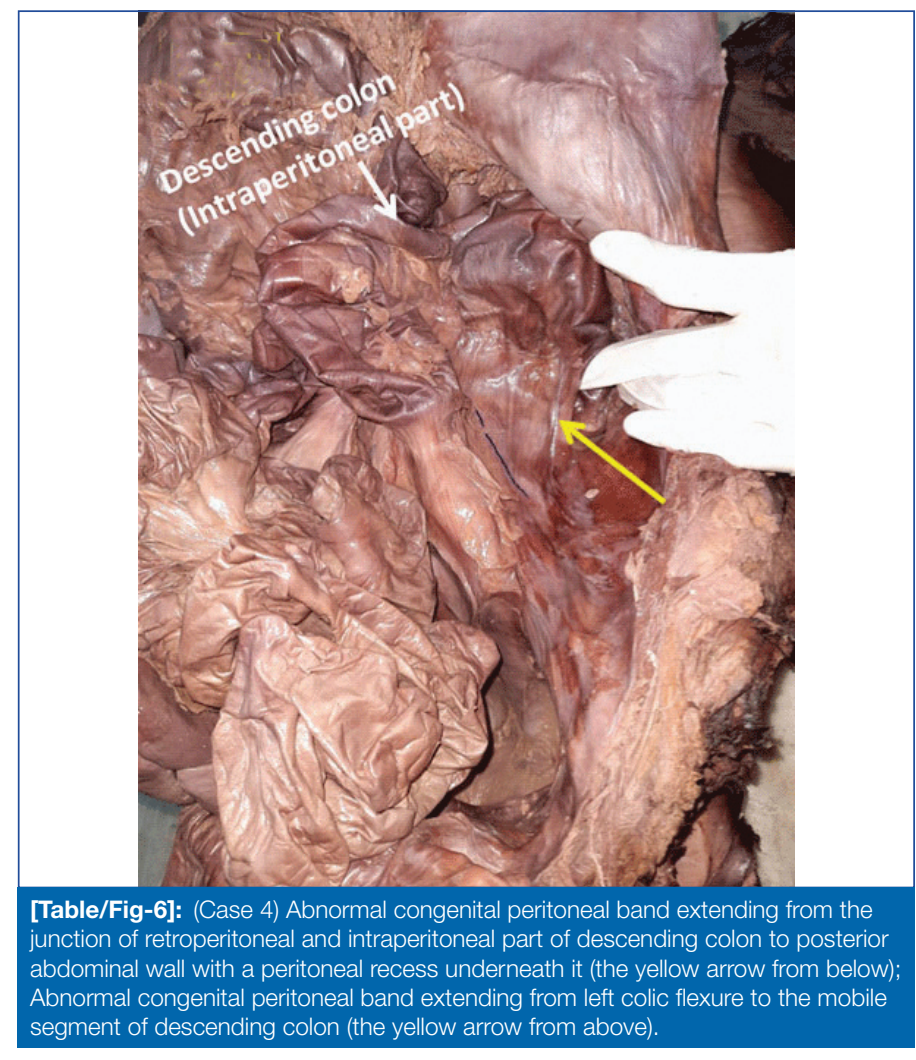

In the above cases, the peritoneal bands are produced during development of gut in fetal life either due to error in any of the three stages of rotation or due to prolongation of attachment of ventral mesogastrium beyond the second part of duodenum. But there are number of reports on intestinal obstruction due to abnormal peritoneal bands without any associated congenital abnormalities of gut. Sannananja B et al., have reported a congenital vascular peritoneal band $30 \mathrm{~cm}$ proximal to the ileocolic junction in the antimesenteric border causing intestinal obstruction in a patient of abdominal tuberculosis [14]. Erginel B et al., in their study on 14 cases of intestinal obstruction due to congenital peritoneal bands, have reported that they joined adjacent parts of intestine, the most common being, terminal ilium to ascending colon (50\%). Others were ilium to ilium, jejunum to jejunum, jejunum to ilium. As the location varies in every patient Erginel $B$ et al., do not agree with the opinion that these congenital bands arise from definitive 
embryological structures [7]. Another series of intestinal obstruction due to congenital peritoneal band was reported by Akgur FM et al., where out of eight cases $50 \%$ had peritoneal band extending from terminal ileum to ascending colon [15]. In the fourth case, a similar band connecting adjacent parts of terminal ileum was found.

\section{Limitation(s)}

The smaller sample size due to scarcity of cadavers might be a limiting factor in the present study because of which the incidence was low as compared to other studies.

\section{CONCLUSION(S)}

Most of the abnormal peritoneal bands in the present study were found to be associated with congenital abnormalities of Gl tract involving rotation or fixation of gut. They were also found in isolation, mostly due to the extension of lesser omentum beyond the first part of duodenum resulting in peritoneal bands like cystoduodenal and cystoduodenocolic ligaments. Though many of these abnormal bands are found only in the dissection room or during autopsy without any apparent symptoms during life, they need to be studied in detail, because they may eventually be complicated by intestinal obstruction or internal herniation.

\section{REFERENCES}

[1] Standring S. The anatomical basis of clinical practice. Development of peritoneum and peritoneal cavity. 41 $1^{\text {st }}$ ed. Elsevier, London. 2016:1098-1109

[2] Sharma NA, Sharma A, Garud RS. Rare peritoneal bands and recesses: Incidental findings in a cadaveric dissection. Surg Radiol Anat. 2013;35(4):359-63.
[3] Kim S, Kim TU, Lee JW. The perihepatic space: Comprehensive anatomy and CT features of pathological conditions. Radiographics. 2007;27:129-43.

[4] Le O. Patterns of peritoneal spread of tumor in the abdomenand pelvis. World $J$ Radiol. 2013;5:106-12

[5] Nayak SB, George BM, Mishra S, Shetty SD, Sirasanagandla SR, Padavinangad A. Surgical and radiological importance of a rare cysto-duodeno-colic peritoneal fold. Anatomy and Cell Biology. 2017;50(2):159-61.

[6] Ashaolu JO, Ukwenya VO, Adenowo TK. Cystoduodenal ligament as an abnorma fold and the accompanyinganatomical and clinical implications. Surgical and Radiologic Anatomy. 2011;33(2):171-74.

[7] Erginel B, Soysal FG, Ozbey H, Keskin E, Celik A, Karadag AJ, et al. Small bowel obstruction due to anomalous congenital bands in children. Gastroenterology Research and Practice. 2016| Article ID 7364329| https://doi.org/10.1155/2016/7364329| Article ID 7364329| https://doi. org/10.1155/2016/7364329.

[8] Bannister LH, Standring SM et al. The anatomical basis of Medicine and Surgery. In Gray's Anatomy. 38 ${ }^{\text {th }}$ (ed.). London, Churchill Livingstone. 1995:1774.

[9] Nayak SB, George BM, Mishra S, Ashwini LS, Marpalli S. Omento-cystic peritoneal fold and rudimentary quadrate lobe: a case report. OA Case Reports. 2013;2(5):46.

[10] Wakefield EG, Mayo CW. Intestinal obstruction produced by mesenteric bands: In association with failure of intestinal rotation. Arch Surg. 1936:33:47.

[11] Appaji AC, Kulkarni R, Kadaba JS. Nonrotation of Intestine: A Case Report. J Clin Diagn Res. 2013;7(11):2575-76.

[12] Hollinshead WH. Anatomy for surgeons. The abdominal viscera and peritoneum. Hober-Harper international edition. Newyork; First reprint edition. 1966;2:287-96.

[13] Saxena P. Nonrotation of midgut loop-a rare cause of recurrent intestina obstruction in adults: case report and review of literature. The Internet Journal of Surgery. 2009;26(1)01-07.

[14] Sannananja B, Shah HU, Avhad G, Agrawal A. Unusual case of an anomalous congenital band causing acute small bowel obstruction in a case of abdominal tuberculosis. J Mahatma Gandhi Inst Med Sci. 2016;21:82-83.

[15] Akgür FM, Tanyel FC, Büyükpamukçu N, Hiçsönmez A. Anomalous congenita bands causing intestinal" obstruction in children. Journal of Pediatric Surgery. 1992:27(4):471-73.

\section{PARTICULARS OF CONTRIBUTORS:}

1. Associate Professor, Department of Anatomy, VIMSAR, Burla, Odisha, India.

2. Assistant Professor, Department of Anatomy, VIMSAR, Burla, Odisha, India

3. Assistant Professor, Department of Anatomy, VIMSAR, Burla, Odisha, India

4. Associate Professor, Department of Anatomy, VIMSAR, Burla, Odisha, India.

\section{NAME, ADDRESS, E-MAIL ID OF THE CORRESPONDING AUTHOR:}

Dr. Srikanta Kumar Mishra,

Associate Professor, Department of Anatomy, VIMSAR, Burla, Odisha, India.

E-mail: srikantamishra.anatomy@gmail.com
PLAGIARISM CHECKING METHODS: [Jain H et al.]

- Plagiarism X-checker: Oct 31, 2020

- Manual Googling: Jan 21, 2021

- iThenticate Software: Mar 04, 2021 (9\%)

\section{AUTHOR DECLARATION:}

- Financial or Other Competing Interests: None

- Was Ethics Committee Approval obtained for this study? Yes

- Was informed consent obtained from the subjects involved in the study? NA

- For any images presented appropriate consent has been obtained from the subjects. NA

Date of Submission: Oct 28, 2020 Date of Peer Review: Dec 23, 2020

Date of Acceptance: Jan 22, 2021

Date of Publishing: Apr 01, 2021 